\title{
A Web-Based Tool to Automate Portions of Pretest Genetic Counseling for Inherited Cancer
}

\author{
Deborah Cragun, $\mathrm{PhD}^{1}$; Anne Weidner, $\mathrm{MPH}^{2}$; Ann Tezak, MA, MPH${ }^{2}$; Brenda Zuniga, $\mathrm{MS}^{2}$; \\ Georgia L. Wiesner, $\mathrm{MD}^{2,3}$; and Tuya Pal, $\mathrm{MD}^{2,3}$
}

\begin{abstract}
Background: Increasing demand for genetic testing for inherited cancer risk coupled with a shortage of providers trained in genetics highlight the potential for automated tools embedded in the clinic process to meet this demand. We developed and tested a scalable, easy-to-use, 12-minute web-based educational tool that included standard pretest genetic counseling elements related to panelbased testing for multiple genes associated with cancer risk. Methods: The tool was viewed by new patients at the Vanderbilt Hereditary Cancer Clinic before meeting with a board-certified genetics professional. Pre- and post-tool surveys measured knowledge, feeling informed/empowered to decide about testing, attitudinal values about genetic testing, and health literacy. Of the initial 100 participants, 50 were randomized to only have knowledge measured on the post-tool survey to assess for a priming effect. Results: Of 360 patients approached, 305 consented and completed both the pre- and post-tool surveys, with a mean age of 47 years, including $80 \%$ female patients and $48 \%$ patients with cancer. Survey results showed an increase in knowledge and feeling informed/empowered after viewing the tool $(P<.001)$, but no significant change in attitude $(P=.64)$. Posttool survey data indicated no difference in median knowledge between low and high health literacy groups $(P=.30)$. No priming effect was present among the initial 100 participants $(P=.675)$. Conclusions: Viewing the educational tool resulted in significant gains in knowledge across health literacy levels, and most individuals felt informed and empowered to decide about genetic testing. These findings indicate that the use of an automated pretest genetic counseling tool may help streamline the delivery of genetic services.
\end{abstract}

J Natl Compr Canc Netw 2020;18(7):841-847 doi: $10.6004 /$ jnccn.2020.7546

${ }^{1}$ College of Public Health, University of South Florida, Tampa, Florida; and ${ }^{2}$ Division of Genetic Medicine, Department of Medicine, Vanderbilt University Medical Center, and ${ }^{3}$ Vanderbilt-Ingram Cancer Center, Nashville, Tennessee.

\section{Background}

Despite the long-standing availability of genetic testing for inherited mutations, including those in BRCAl and $B R C A 2,<20 \%$ of patients with breast or ovarian cancer meeting national criteria for hereditary cancer susceptibility receive testing. ${ }^{1}$ Identification of inherited cancer risk empowers individuals and their families to detect cancers at the earliest stages or prevent them altogether, and is increasingly being used to guide treatment. ${ }^{2-11}$ The coupling of genetic testing for hereditary cancer risk with pretest and posttest genetic counseling (GC) is endorsed by several national organizations $\mathrm{s}^{12-15}$ and is a requirement for a breast center to become accredited. ${ }^{16}$ Since 1996 ASCO has provided guidance on standard elements to be discussed during the pretest GC session, ${ }^{17}$ conducted by certified genetics health professionals (GHPs) ${ }^{18}$ such as genetic counselors, clinical geneticists, or other appropriately trained healthcare providers.

Given an upsurge of testing, a shortage of GHPs, and a healthcare workforce with limited proficiency in genetics, ${ }^{19}$ most genetic testing for inherited cancer is performed by those without formal training in genetics, and often using limited or no pretest GC. ${ }^{20-22}$ Yet policies that mandate pretest GC through a GHP may disproportionately reduce testing rates among underserved populations. ${ }^{23,24}$ Consequently, new streamlined models to offer effective pretest and posttest GC services across all at-risk populations are needed.

Although automated tools for pretest GC have previously shown promise, most prior efforts focused solely on BRCA testing. ${ }^{25-27}$ Since the implementation of multigene panel testing, a single publication examined an automated tool (through a video) for pretest GC among patients with ovarian cancer. ${ }^{28}$ Results indicated higher testing rates among those who saw the video compared with those who received in-person GC, presumably because it was convenient and did not require a separate visit. ${ }^{28}$ However, the study did not collect data to evaluate for increases in knowledge or other patient-reported outcomes; thus, it remains unknown whether the video was sufficient at ensuring patients were knowledgeable 
and felt informed and empowered to decide and whether the decision they ultimately made was concordant with their attitudinal values, which are cited as important components of an informed decision. ${ }^{29,30}$ Given that the use of automated tools to conduct pretest GC may replace components of an existing billable clinical service, it is critical to evaluate and test such tools before their widespread use.

Through the proposed study, we sought to develop and test a web-based educational tool to cover pretest GC elements for multigene panel testing and measure the impact of the tool on patients' (1) knowledge, (2) feeling informed/empowered to decide, and (3) attitudinal values about testing. We also sought to evaluate the relationship between health literacy and these outcomes.

\section{Methods}

\section{Educational Tool Development}

A 12-minute web-based educational tool was developed to broadly cover standard elements discussed in a pretest GC session for inherited cancer predisposition, including expanded testing through multigene panels as outlined in the 2016 ASCO guidelines, ${ }^{13}$ and did not focus on any specific syndrome or organ site. Through an iterative process, input was collected from several GHPs, including genetic counselors, clinical geneticists, genetics-certified nurses, and GC students, and was used to refine the content, images, and script. The educational tool was subsequently narrated with animations using Articulate software for listeners to view at their own pace and return to slides they might want to review again.

\section{Survey Development and Measures}

Surveys measured 3 constructs: (1) knowledge about hereditary cancer testing, (2) feeling informed and empowered to decide about testing measured through the SURE (Sure of myself, Understand information, Risk-benefit ratio, Encouragement) checklist (ie, the opposite of decisional conflict), ${ }^{31}$ and (3) attitudes measuring the extent to which participants valued genetic testing for hereditary cancer. An additional question was included as a measure of health literacy. ${ }^{32}$

Knowledge questions were developed to assess patients' understanding of standard elements addressed during a pretest GC session focused on inherited cancer and aligned with the 2016 ASCO guidelines inclusive of multigene panel testing content. ${ }^{13}$ At the time of this study, no existing knowledge scales covered multigene panel testing (not limited to specific hereditary cancers) that included pretest elements recommended per ASCO guidelines. ${ }^{13,33}$ Furthermore, newer knowledge scales developed for whole-exome or genome testing were too broad to measure knowledge gains focused specifically on inherited cancer predisposition. ${ }^{34}$

Specifically, 35 questions were developed and anonymously tested among 445 individuals who completed the questions online through postings and emails using various listservs, registries, and social media platforms (unpublished data). Psychometric analyses were conducted and written feedback about the questions was reviewed to refine the questions. The number of questions was then reduced to 10 to cover standard elements of the GC session per the key concepts from the ASCO guidelines $^{13}$ : inheritance, possible test results, use of test results for cancer prevention and risk management, and potential limitations of genetic testing, including the extent to which laws protect against genetic discrimination. Response options for each item included "agree," "disagree," and "don't know." Possible knowledge scores could range from 0 to 10 , with 1 point assigned for each correct answer.

The previously validated 4 -item SURE checklist $^{31}$ was used with the response options of "yes," "no," and "unsure." Participants who answered yes to all 4 SURE checklist items were classified as feeling informed and empowered to decide about genetic testing, while all others were classified as having at least some degree of decisional conflict about genetic testing.

Attitudinal values about genetic testing were collected through 3 Likert-scale items developed to assess the extent to which individuals wanted genetic testing and perceived it to be useful and important. These items showed high inter-item reliability (Cronbach $\alpha=0.94)$ and were reversecoded and averaged to create an "attitudinal values" score ranging from 1 to 5 . Scores were then dichotomized such that an average score of $\geq 4$ constituted a favorable attitude toward genetic testing.

The single-item question, "How often do you have problems learning about a medical condition because of difficulty understanding written information?" was included as a proxy measure for health literacy because, when pretested with 445 individuals alongside the knowledge questions, it correlated more strongly with knowledge than 2 other potential health literacy screening questions from a previously validated measure. ${ }^{32}$ The 5 response options were dichotomized such that individuals indicating problems some, most, or all of the time were combined into a low-literacy group, whereas those with problems only a little of the time or never were categorized into a high-literacy group.

\section{Participant Recruitment and Data Collection}

After Institutional Review Board approval through Vanderbilt University, the web-based educational tool was tested among patients who were scheduled for a new patient in-person appointment for their first GC visit at the Vanderbilt Hereditary Cancer Clinic, a single clinic 
based at an academic medical center. Patients with a personal and/or family history of various cancers are referred by their provider or self-refer to this clinic for cancer genetic risk assessment and GC or testing for any type of cancer-related condition. Patients were seen by GHPs staffing the clinic, including 2 board-certified clinical geneticists, 2 board-certified genetic counselors, and 1 nurse practitioner certified in genetics. Eligible patients were aged $\geq 18$ years, spoke English, consented to participate in the study, and completed online surveys before and after viewing the tool. Patients who underwent prior genetic testing, but did not receive GC through a certified or credentialed genetics professional, were eligible for participation. Consent, completion of the surveys, and viewing of the educational tool were all webbased and administered via iPad in either the waiting area or the clinic room before the patient met with the GHP.

To evaluate for a priming effect of the knowledge questions, the first 100 participants were randomly assigned to either group A or B using block randomization with block sizes between 1 and 5 individuals. ${ }^{35}$ Participants in both groups were directed to complete an online survey before viewing the web-based educational tool (the pre-tool questionnaire), which included questions assessing informed/empowered choice, attitudinal values, and health literacy. In addition, those in group A were asked the knowledge questions on the pre-tool questionnaire to determine whether seeing the knowledge questions before viewing the web-based educational tool led to a priming effect. After completing the pre-tool questionnaire, both groups were automatically directed to view the tool and then immediately prompted to complete an online follow-up survey (the post-tool questionnaire). The post-tool questionnaire was the same across both groups and included questions on knowledge, feeling informed/empowered to decide, attitudinal values, and 3 open-ended questions eliciting feedback about the tool. After recruitment of the first 100 participants, all subsequent participants were recruited to group A. With consent from each participant, demographic and clinical data including sex, race/ethnicity, marital status, insurance, parity, personal and family history of cancer, and previous genetic testing were collected from patient records.

\section{Data Analysis}

Median pre-tool and post-tool questionnaire knowledge scores among participants in group A were calculated and assessed using the Wilcoxon signed-rank test. Knowledge changes were also assessed using the Wilcoxon signed-rank test. Median knowledge scores were also calculated for patients with and without (1) previous genetic testing, (2) a personal history of cancer, (3) a family member with a positive (ie, pathogenic or likely pathogenic) genetic test result, and (4) high health literacy.
Independent-samples median tests assessed for knowledge score differences across these groups, and significance was determined after using the Holm-Bonferroni method to control for the family-wise error rates.

The proportion of participants who felt fully informed and empowered to make a decision (ie, answered yes to all 4 SURE items) and the proportion who were favorable toward genetic testing (ie, averaged $\geq 4$ on the attitudinal values scale) were calculated. Changes in whether participants felt informed/empowered and held favorable attitudes toward genetic testing before and after viewing the tool were evaluated using McNemar tests. Differences in feeling informed and empowered to decide and differences in attitudinal values were compared across patients with and without (1) previous genetic testing, (2) a personal history of cancer, (3) a family member with a positive (ie, pathogenic or likely pathogenic) genetic test result, and (4) high health literacy using chi-square tests and the Holm-Bonferroni method to control for the family-wise error rates.

The priming effect (ie, whether seeing the knowledge questions before viewing the web-based educational tool increased the knowledge score) was assessed among the initial 100 participants by comparing post-tool questionnaire knowledge scores between groups A and B using an independent-samples median test. Demographic and clinical characteristics were compared across the 2 groups using chi-square tests to confirm equivalency. All statistical tests were conducted using SPSS Statistics (IBM Corp, version 26) and were considered significant at $\alpha=0.05$.

\section{Results}

The study enrolled 321 patients from November 2018 to March 2020. Of the 360 individuals approached, 321 consented, of whom 305 completed the pre-tool and post-tool questionnaires and were included in the analysis: 255 were assigned to group A and 50 to group B. Mean age was 47 years, $80 \%$ of patients were female, and $48 \%$ had a cancer diagnosis (Table 1). Among group A participants, median pre-tool and post-tool knowledge scores increased from 3 to 8 ( $P<.001$; Table 2$)$. Differences in median pre-tool and post-tool knowledge scores based on those with and without (1) previous genetic testing, (2) a personal history of cancer, and (3) a family member with a positive genetic test result were not significant $(P$ values $>.05)$, other than for pre-test knowledge scores among individuals with versus without prior testing $(P=.001)$.

Median pre-tool knowledge scores among low and high health literacy groups were 2 and 4, respectively $(P=.06)$. This gap narrowed after participants viewed the tool, with post-tool knowledge scores of 8 and 8 , respectively $(P=.30)$.

As summarized in Table 3 , only $29 \%$ of participants (ie, 87 of 305) felt fully informed and empowered to make 


\begin{tabular}{|c|c|}
\hline & n (\%) \\
\hline Total & $305(100)$ \\
\hline High health literacy & $231(76)$ \\
\hline Female & $244(80)$ \\
\hline Non-Hispanic white ${ }^{a}$ & $257(86)$ \\
\hline Married or cohabitating ${ }^{b}$ & $216(74)$ \\
\hline Private insurance & $232(76)$ \\
\hline Has children & $234(77)$ \\
\hline Personal history of cancer & $147(48)$ \\
\hline Had previous genetic testing & $38(12)$ \\
\hline Family member with positive result $t^{c}$ & $52(17)$ \\
\hline Family history of cancer ${ }^{c, d}$ & $266(88)$ \\
\hline
\end{tabular}

aExcludes 5 unknowns; overall percent reported is out of $n=300$.

bexcludes 13 unknowns; overall percent reported is out of $n=292$.

'Excludes 1 unknown; overall percent reported is out of $n=304$.

Includes breast, ovarian, endometrial, prostate, colorectal, or pancreatic cancer.

a decision about genetic testing before viewing the tool, which increased to $74 \%$ (ie, 227 of 305) after viewing the tool $(P<.001)$. The proportion of participants with favorable attitudinal values toward genetic testing remained similar before and after viewing the tool ( $82 \%$ vs $83 \%$; $P=.64$; Table 3 ). There were no significant differences in the proportion of those who felt fully informed/empowered or those who held favorable attitudinal values based on (1) previous genetic testing, (2) a personal history of cancer, and (3) a family member with a positive (ie, pathogenic or likely pathogenic) genetic test result (all $P>.05$ ). However, those with high health literacy were significantly more likely than those with low health literacy to report being fully informed and empowered to make a decision both before (35\% vs $8 \% ; P<.001)$ and after (79\% vs $61 \% ; P=.002)$ viewing the tool. Similarly, a larger percentage of those with high health literacy had favorable attitudes toward genetic testing both before $(85 \%$ vs $73 \% ; P=.025)$ and after ( $85 \%$ vs $77 \%$; $P=.108$ ) viewing the tool.

Among the first 100 participants, post-tool knowledge scores assessed between groups A and B were similar $(P=.675)$, and there were no significant differences in clinical or demographic characteristics (data not shown), suggesting no priming effect.

\section{Discussion}

This is the first study to measure multiple patientreported outcomes after viewing an electronic pretest GC educational tool focused on inherited cancer risk, including content about multigene panel tests. The webbased educational tool, which was designed to inform individuals about genetic testing rather than persuade them to have testing, showed minimal impact on attitudinal values, resulted in significant gains in knowledge pertaining to pretest GC elements, ${ }^{13}$ and seemed to close the knowledge gap between those with low and high health literacy levels, and most participants felt informed and empowered to make a decision about testing after viewing the tool. These findings support the potential to automate components of pretest GC to streamline the delivery of GC and testing services for inherited cancer risk across broad populations.

As demand and indications for genetic testing expand and encompass treatment indications at point of care, ${ }^{9,10,36-47}$ maintaining quality while developing strategies to scale up services is critical. Although we recognize that in-person pretest GC is considered "usual care," most patients are tested by healthcare providers with no formal training in genetics, and receive limited or no pretest GC. ${ }^{20-22}$ Access to GHPs is especially low in rural areas, ${ }^{48,49}$ certain states, and community oncology practices. ${ }^{50-52}$ Our educational tool has promise for filling this gap given that it is scalable and uses fewer resources than in-person services. Based on our results, we believe the tool could increase access to pretest GC knowledge while enabling most patients (regardless of health literacy level) to feel informed and empowered to make a decision about testing. This would preserve GHP availability for individuals who may benefit most from pretest GC and allow GHPs to focus on posttest results disclosure, interpretation, and guidance about followup care, where they may have the most potential to enhance patient care.

Despite the potential value of this educational tool, we recognize that some individuals may still benefit from pretest GC through a GHP or other healthcare provider. The multiple parameters measured in our study may serve as the basis to develop a system to triage those who may benefit from pretest GC, such as those who score low on knowledge or do not feel fully informed and empowered to make a decision about genetic testing after viewing the educational tool. Our finding that more individuals with low health literacy continued to have some level of decisional conflict suggests that education alone may be insufficient to empower genetic testing decision-making among some individuals and that this may be an important area of focus in GC sessions.

Although it remains important to streamline pretest GC services for inherited cancer, which was the focus of our current study, it is also important to consider other components that need to be addressed, including creating a 3- or 4-generation pedigree through which a comprehensive risk assessment may be performed and ordering the actual genetic testing based on indications. Consequently, there remains a need to automate the collection of family history to streamline delivery of these 


\section{Table 2. Participants Who Answered Each Knowledge Question Correctly}

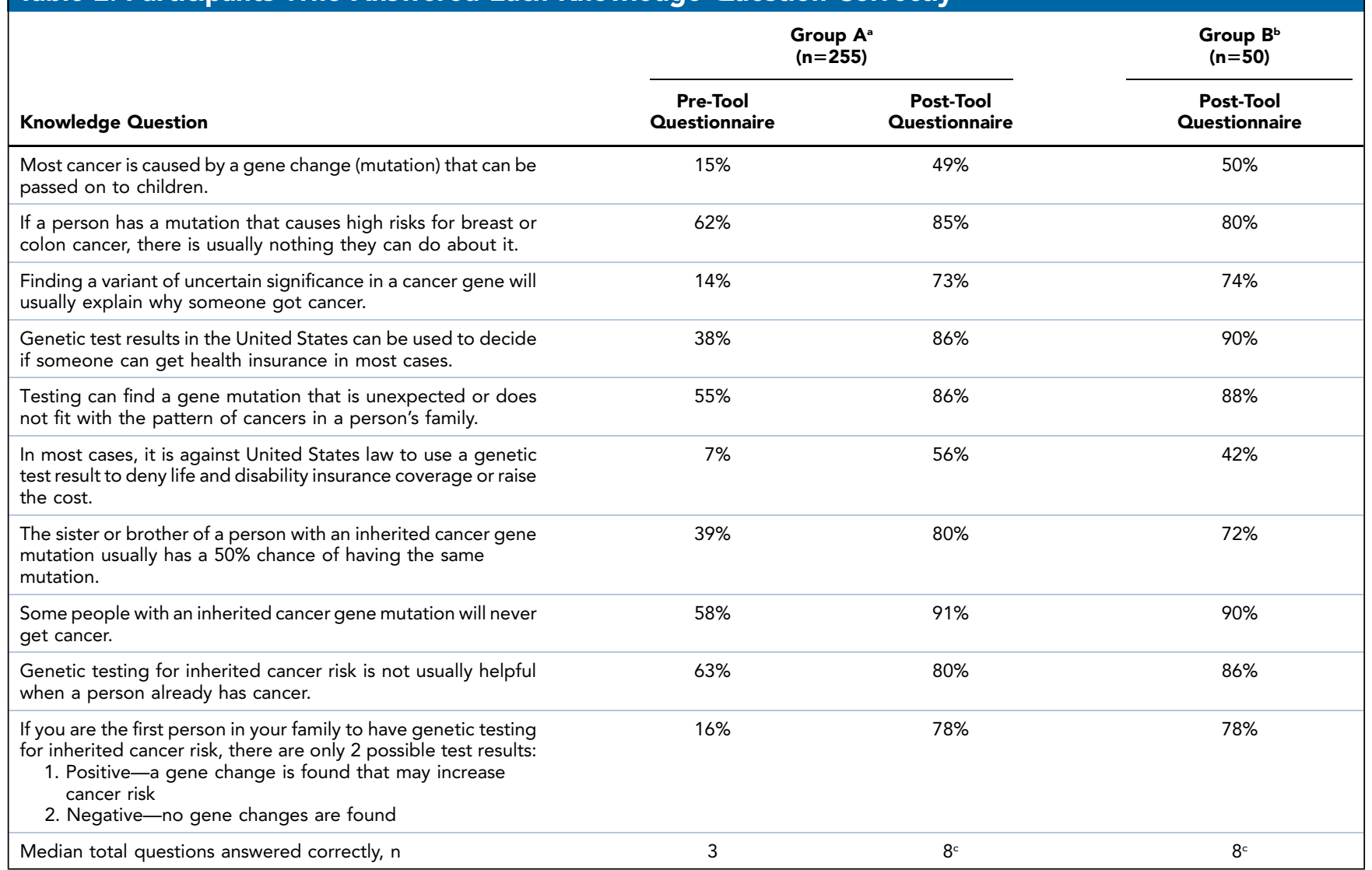

aGroup A completed knowledge questions before and after viewing the tool and showed a statistically significant increase in median knowledge $(P<.001)$. ${ }^{b}$ Group $B$ completed knowledge questions only after viewing the tool to assess for a priming effect.

'No priming effect was identified based on the lack of significant difference in median post-tool knowledge scores among the initial 100 participants across groups $A$ and $B(P=.675)$.

services, and GHPs are critical in setting up processes to ensure that testing is clinically indicated and that results are interpreted within the context of family history. Furthermore, patients may have other psychosocial needs that will be unmet with increased automation, and it is important to recognize that informed consent is only one of several goals of GC.
The current study has several strengths, including a wellcharacterized sample with a clinical population presenting with diverse indications for risk assessment and GC for inherited cancer. Furthermore, our study design enabled us to alleviate concern that the knowledge increases occurred because of a priming effect resulting from administering the knowledge questions on the pre-tool questionnaire.

\section{Table 3. Participant Response After Viewing the Web-Based Educational Tool}

\begin{tabular}{|c|c|c|c|c|}
\hline & & & \multicolumn{2}{|c|}{$\begin{array}{c}\text { After Viewing (Post-Tool } \\
\text { Questionnaire) }\end{array}$} \\
\hline & & & $\begin{array}{c}\text { No } \\
n(\%)\end{array}$ & $\begin{array}{c}\text { Yes } \\
\text { n (\%) }\end{array}$ \\
\hline \multirow[t]{2}{*}{ Felt informed/empowered ${ }^{a}$} & Before viewing (pre-tool questionnaire) & No & $72(24)$ & $146(48)$ \\
\hline & & Yes & $6(2)$ & $81(27)$ \\
\hline Had favorable attitudinal values ${ }^{b}$ & Before viewing (pre-tool questionnaire) & No & $32(10)$ & $23(8)$ \\
\hline
\end{tabular}

aMcNemar test identified a significant change $(P<.001)$.

${ }^{\mathrm{b}} \mathrm{McN}$ emar test found no significant change $(P=.643)$. 
Finally, our GC educational tool was developed to address required elements per the 2016 ASCO guidelines. ${ }^{13}$

Despite these strengths, we recognize a number of limitations, including use of a new knowledge scale that would benefit from additional validity testing to ensure that it addresses the required content. Validated questions measuring content of pretest GC education focused on inherited cancer testing with incorporation of multigene panel tests were only recently published, after our study was completed, and those questions seem to cover similar content as our questions. ${ }^{53}$ Moreover, the SURE checklist, ${ }^{31}$ which has not been previously used in this context, was chosen because of its brevity and based on a recent GC outcomes framework outlining the spectrum ranging from decisional conflict to feeling informed and empowered to make a decision. ${ }^{54}$ Although the SURE checklist was sensitive to change in this study, it remains important to evaluate it in additional studies. Although more comprehensive or nuanced measures of informed consent and health literacy exist, there remains a lack of consensus in the genetics community about what constitutes informed consent in the context of genetic testing in the clinical setting. ${ }^{55}$ Furthermore, we chose to use brief measures to increase our ability to implement a survey in the context of a busy clinical service. The brevity of our survey, while increasing feasibility, may be less reliable than more lengthy measures. In addition, our study may not be generalizable to all settings given that it was conducted at a single academic site within an insured, mostly non-Hispanic white population with limited diversity. Specifically, the favorable baseline attitudinal scores may be related to characteristics of individuals in this study population who were already scheduled for a cancer genetic risk assessment. More research is needed to examine the utility of a pretest GC tool among minority and underserved populations, which encompasses the focus of our ongoing efforts. Finally, our study was not designed to measure the impact of the educational tool on the subsequent GC process or informed decisionmaking through measurement of uptake of testing.

Although the results of our web-based tool are promising, future efforts are needed to compare these types of automated interventions versus standard-of-care GC, ideally through a hybrid study encompassing a randomized trial alongside a collection of implementation factors, including measurement of efficiency and satisfaction. It is also important to consider implementing this tool in the patient care workflow and incorporating other components performed during genetic risk assessment, including generation of a pedigree, collection of personal and family history, and completion of informed consent for testing. It is conceivable that workflow would incorporate specific discussion items into a posttest GC session when results are disclosed. The provider could then review the personal and family history, with results in hand, to inform risk assessment, additional testing considerations, and recommended management.

\section{Conclusions}

Findings from our study indicate that a web-based pretest GC tool for inherited cancer predisposition significantly increases knowledge based on the scale created for this effort, and suggest that among individuals who presented to a hereditary cancer clinic, most felt informed and empowered to make a decision about genetic testing. These efforts are critical as the healthcare system seeks strategies to scale up and streamline the delivery of cancer genetic risk assessment and counseling services while maximizing the benefits of tailored posttest GC discussion, including results interpretation and guidance on medical management.

\section{Acknowledgments}

We thank Courtney Lewis for her efforts in the initial version of the tool, and Joy Kechik and other genetic counseling students at the University of South Florida Genetic Counseling Graduate Program. We thank Carlos Montoya and the University of South Florida College of Public Health ETA office for technical assistance with the software used in the development of the tool.

Submitted November 5, 2019; accepted for publication February 7, 2020.

Author contributions: Study concept: Cragun, Pal. Methodology: Cragun, Pal. Project administration: Weidner, Tezak. Data curation: Weidner. Formal analysis: Cragun, Weidner. Investigation: Zuniga. Funding acquisition: Pal. Writing -original draft: Cragun. Writing_review and editing: All authors.

Disclosures: The authors have disclosed that they have not received any financial consideration from any person or organization to support the preparation, analysis, results, or discussion of this article.

Funding: This work was supported by funding from Ingram Professorship (ID0EQ6AG3405), Kleberg Foundation (IDOESDBG3406), and Vanderbilt Genetic Institute Departmental Funds (IDOEUHBG3407). This project was also supported by CTSA (award number UL1 TR002243) from the National Center for Advancing Translational Sciences. Its contents are solely the responsibility of the authors and do not necessarily represent official views of the National Center for Advancing Translational Sciences or the NIH.

Correspondence: Tuya Pal, MD, Vanderbilt-Ingram Cancer Center, 1500 21st Avenue South, Suite 2810, Nashville, TN 37212. Email: tuya.pal@vumc.org

\section{References}

1. Childers $\mathrm{CP}$, Childers KK, Maggard-Gibbons M, et al. National estimates of genetic testing in women with a history of breast or ovarian cancer. $\mathrm{J}$ Clin Oncol 2017;35:3800-3806.

2. Watson M, Kash KM, Homewood J, et al. Does genetic counseling have any impact on management of breast cancer risk? Genet Test 2005;9:167-174.

3. Roukos $\mathrm{DH}$, Briasoulis $\mathrm{E}$. Individualized preventive and therapeutic management of hereditary breast ovarian cancer syndrome. Nat Clin Pract Oncol 2007;4:578-590.

4. Narod SA, Offit K. Prevention and management of hereditary breast cancer. J Clin Oncol 2005;23:1656-1663. 
5. Domchek SM, Friebel TM, Singer CF, et al. Association of risk-reducing surgery in BRCA1 or BRCA2 mutation carriers with cancer risk and mortality. JAMA 2010;304:967-975.

6. Finch AP, Lubinski J, Møller P, et al. Impact of oophorectomy on cancer incidence and mortality in women with a BRCA1 or BRCA2 mutation. $\mathrm{J}$ Clin Oncol 2014;32:1547-1553.

7. Maxwell KN, Domchek SM. Cancer treatment according to BRCA1 and BRCA2 mutations. Nat Rev Clin Oncol 2012;9:520-528.

8. Tinoco G, Warsch S, Glück $S$, et al. Treating breast cancer in the 21 st century: emerging biological therapies. J Cancer 2013;4:117-132.

9. Robson M, Im SA, Senkus E, et al. Olaparib for metastatic breast cancer in patients with a germline BRCA mutation. N Engl J Med 2017;377:523-533.

10. Le DT, Uram JN, Wang H, et al. PD-1 blockade in tumors with mismatchrepair deficiency. N Engl J Med 2015;372:2509-2520.

11. Westdorp $\mathrm{H}$, Kolders $\mathrm{S}$, Hoogerbrugge $\mathrm{N}$, et al. Immunotherapy holds the key to cancer treatment and prevention in constitutional mismatch repair deficiency (CMMRD) syndrome. Cancer Lett 2017;403:159-164.

12. Hereditary Cancer Syndromes and Risk Assessment: ACOG Committee Opinion, Number 793. Obstet Gynecol 2019;134:e143-149.

13. Robson ME, Bradbury AR, Arun B, et al. American Society of Clinical Oncology policy statement update: genetic and genomic testing for cancer susceptibility. J Clin Oncol 2015;33:3660-3667.

14. Owens DK, Davidson KW, Krist AH, et al. Risk assessment, genetic counseling, and genetic testing for BRCA-related cancer: US Preventive Services Task Force recommendation statement. JAMA 2019;322:652-665.

15. Daly MB, Pilarski R Perry MP, et al. NCCN Clinical Practice Guidelines in Oncology: Genetic/Familial High-Risk Assessment: Breast, Ovarian, and Pancreatic. Version 1.2020. Accessed December 4, 2019. To view the most recent version, visit NCCN.org.

16. American College of Surgeons. National Accreditation Program for Breast Centers Standards Manual, 2nd ed. Accessed June 15, 2019. Available at: https://accreditation.facs.org/accreditationdocuments/NAPBC/Portal\% 20Resources/2018NAPBCStandardsManual.pdf

17. American Society of Clinical Oncology. Statement of the American Society of Clinical Oncology: genetic testing for cancer susceptibility, adopted on February 20, 1996. J Clin Oncol 1996;14:1730-1736 [discussion: 1737-1740].

18. Hoskovec JM, Bennett RL, Carey ME, et al. Projecting the supply and demand for certified genetic counselors: a workforce study. J Genet Couns 2018;27:16-20.

19. Rubanovich CK, Cheung C, Mandel J, et al. Physician preparedness for big genomic data: a review of genomic medicine education initiatives in the United States. Hum Mol Genet 2018;27:R250-258.

20. Cragun D, Camperlengo L, Robinson E, et al. Differences in BRCA counseling and testing practices based on ordering provider type. Genet Med 2015;17:51-57.

21. Vadaparampil ST, Scherr CL, Cragun D, et al. Pretest genetic counseling services for hereditary breast and ovarian cancer delivered by non-genetics professionals in the state of Florida. Clin Genet 2015; 87:473-477.

22. Katz SJ, Ward KC, Hamilton AS, et al. Gaps in receipt of clinically indicated genetic counseling after diagnosis of breast cancer. J Clin Oncol 2018;36: 1218-1224.

23. Whitworth $P$, Beitsch $P$, Arnell $C$, et al. Impact of payer constraints on access to genetic testing. J Oncol Pract 2017;13:e47-56.

24. Stenehjem DD, Au T, Sainski AM, et al. Impact of a genetic counseling requirement prior to genetic testing. BMC Health Serv Res 2018;18:165.

25. Green MJ, Peterson SK, Baker MW, et al. Effect of a computer-based decision aid on knowledge, perceptions, and intentions about genetic testing for breast cancer susceptibility: a randomized controlled trial. JAMA 2004;292:442-452.

26. Sie AS, van Zelst-Stams WA, Spruijt L, et al. More breast cancer patients prefer BRCA-mutation testing without prior face-to-face genetic counseling. Fam Cancer 2014;13:143-151.

27. Manchanda R, Burnell M, Loggenberg K, et al. Cluster-randomised non-inferiority trial comparing DVD-assisted and traditional genetic counselling in systematic population testing for BRCA1/2 mutations. J Med Genet 2016:53:472-480.

28. Watson $\mathrm{CH}$, Ulm $\mathrm{M}$, Blackburn $\mathrm{P}$, et al. Video-assisted genetic counseling in patients with ovarian, fallopian and peritoneal carcinoma. Gynecol Oncol 2016;143:109-112.

29. Ames AG, Metcalfe SA, Dalton Archibald A, et al. Measuring informed choice in population-based reproductive genetic screening: a systematic review. Eur J Hum Genet 2015;23:8-21.

30. Sepucha K, Ozanne E, Silvia K, et al. An approach to measuring the quality of breast cancer decisions. Patient Educ Couns 2007;65:261-269.
31. Ferron Parayre A, Labrecque M, Rousseau M, et al. Validation of SURE, a four-item clinical checklist for detecting decisional conflict in patients. Med Decis Making 2014;34:54-62.

32. Chew LD, Griffin JM, Partin MR, et al. Validation of screening questions for limited health literacy in a large VA outpatient population. J Gen Intern Med 2008:23:561-566.

33. Furr LA, Kelly SE. The Genetic Knowledge Index: developing a standard measure of genetic knowledge. Genet Test 1999;3:193-199.

34. Kaphingst KA, Facio FM, Cheng MR, et al. Effects of informed consent for individual genome sequencing on relevant knowledge. Clin Genet 2012; 82:408-415.

35. REDCap Randomization Module. Accessed June 30, 2018. Available at: https://wiki.uiowa.edu/display/REDCapDocs/REDCap+Randomization+ Module

36. Ashworth A, Lord CJ. Synthetic lethal therapies for cancer: what's next after PARP inhibitors? Nat Rev Clin Oncol 2018;15:564-576.

37. Kamel D, Gray C, Walia JS, et al. PARP inhibitor drugs in the treatment of breast, ovarian, prostate and pancreatic cancers: an update of clinical trials. Curr Drug Targets 2018;19:21-37.

38. Le D, Gelmon KA. Olaparib tablets for the treatment of germ line BRCA-mutated metastatic breast cancer. Expert Rev Clin Pharmacol 2018 11:833-839.

39. Ledermann JA, Harter P, Gourley $C$, et al. Overall survival in patients with platinum-sensitive recurrent serous ovarian cancer receiving olaparib maintenance monotherapy: an updated analysis from a randomised, placebocontrolled, double-blind, phase 2 trial. Lancet Oncol 2016;17:1579-1589.

40. Link JT, Overman MJ. Immunotherapy progress in mismatch repair-deficient colorectal cancer and future therapeutic challenges. Cancer J 2016;22:190-195

41. Litton JK, Rugo HS, Ettl J, et al. Talazoparib in patients with advanced breast cancer and a germline BRCA mutation. N Engl J Med 2018;379: 753-763.

42. Lyons TG, Robson ME. Resurrection of PARP inhibitors in breast cancer. J Natl Compr Canc Netw 2018;16:1150-1156.

43. Overman MJ, Ernstoff MS, Morse MA. Where we stand with immunotherapy in colorectal cancer: deficient mismatch repair, proficient mismatch repair, and toxicity management. Am Soc Clin Oncol Educ Book 2018;38:239-247

44. Overman MJ, Lonardi S, Wong KYM, et al. Durable clinical benefit with nivolumab plus ipilimumab in DNA mismatch repair-deficient/microsatellite instability-high metastatic colorectal cancer. J Clin Oncol 2018;36: 773-779.

45. Ramakrishnan Geethakumari $P$, Schiewer MJ, Knudsen KE, et al. PARP inhibitors in prostate cancer. Curr Treat Options Oncol 2017;18:37.

46. Staropoli N, Ciliberto D, Del Giudice T, et al. The era of PARP inhibitors in ovarian cancer: "class action" or not? A systematic review and meta-analysis. Crit Rev Oncol Hematol 2018;131:83-89.

47. Fong PC, Boss DS, Yap TA, et al. Inhibition of poly(ADP-ribose) polymerase in tumors from BRCA mutation carriers. N Engl J Med 2009;361:123-134.

48. Bookman T. More people seek genetic testing, but there aren't enough counselors. Accessed October 11, 2016. Available at: http://www.npr.org/ sections/health-shots/2016/04/18/473066953/more-people-seekgenetic-testing-but-there-arent-enough-counselors

49. U.S. Bureau of Labor Statistics. Occupational employment and wages, May 2016: 29-9092 Genetic Counselors. Accessed April 21, 2020. Available at: https://www.bls.gov/oes/2016/may/oes299092.htm

50. Acheson LS, Stange KC, Zyzanski S. Clinical genetics issues encountered by family physicians. Genet Med 2005;7:501-508.

51. Vig HS, Armstrong J, Egleston BL, et al. Cancer genetic risk assessment and referral patterns in primary care. Genet Test Mol Biomarkers 2009;13: 735-741.

52. Radford C, Prince A, Lewis K, et al. Factors which impact the delivery of genetic risk assessment services focused on inherited cancer genomics: expanding the role and reach of certified genetics professionals. J Genet Couns 2014;23:522-530.

53. Underhill-Blazey M, Stopfer J, Chittenden A, et al. Development and testing of the KnowGene scale to assess general cancer genetic knowledge related to multigene panel testing. Patient Educ Couns 2019;102:1558-1564.

54. Cragun D, Zierhut H. Development of FOCUS-GC: Framework for Outcomes of Clinical Communication Services in Genetic Counseling. J Genet Couns 2018;27:33-58.

55. Rego S, Grove ME, Cho MK, et al. Informed consent in the genomics era [published online September 30, 2019]. Cold Spring Harb Perspect Med, https://doi.org/10.1101/cshperspect.a036582 